\title{
A educação e o ensino de bioética em época de pandemia
}

\author{
Education and bioethics teaching in times of pandemic
}

\author{
Waldemar Antônio das Neves Júnior ${ }^{1}$ (1) | waldemarneves@hotmail.com \\ Lumaira Maria Nascimento Silva da Rocha Marques' (1) lumaira_@hotmail.com \\ Michelle Cecille Bandeira Teixeira² (D) michelle.cecille@gmail.com
}

\section{RESUMO}

Introdução: Com as atividades presenciais suspensas em decorrência da pandemia, as instituições de ensino superior tiveram de discutir e executar o processo educativo e reinventar-se em relação ao processo educativo a ele, de modo a oferecer o acesso remoto e, ao mesmo tempo, amenizar a exclusão sociodigital.

Desenvolvimento: O objetivo deste artigo é realizar uma reflexão propositiva para o ensino remoto da bioética sob o olhar da educação em valores e justiça social. O perfil socioeconômico dos discentes não pode ser ignorado quando se planeja a educação on-line, fato que impacta diretamente $\mathrm{o}$ acesso às aulas por meio de computadores e internet. $\mathrm{O}$ artigo propõe utilizar as capacidades da inteligência moral como objetivos de aprendizagem, revisar os problemas preexistentes, contextualizá-los à nova realidade dos conteúdos didáticos e refletir sobre como a bioética pode contribuir para as discussões sobre as desigualdades sociais que aumentaram de forma exponencial neste momento de crise.

Conclusão: As reflexões propositivas aqui apresentadas podem ser utilizadas no modelo de ensino remoto, presencial ou híbrido.

Palavras-chave: Ensino; Educação; Bioética; Ensino On-line; Pandemia.

\section{ABSTRACT}

Introduction: Due to the suspension of face-to-face activities during the COVID-19 pandemic, Higher Education Institutions had to discuss and plan alternative actions in an attempt to readequate themselves to emerging educational demands in order to offer remote accessibility to the academic community and, consequently, reduce social and digital exclusion.

Development: Having that in mind, this article aims at offering a reflection on the teaching of bioethics under the perspective of social justice and education. Within this context, the students' socioeconomic profile cannot be ignored in the planning of online education, since it directly affects students' accessibility to academic activities through the use of computers and the internet. Therefore, this article proposes the use of moral intelligence skills as learning goals, in addition to revising and contextualizing pre-existing problems prior to the new reality of didactic contents. Moreover, it proposes a reflection on how bioethics may contribute to the discussions on the increase in social inequalities during this moment of crisis.

Conclusion: The reflections presented in this article can be used in both remote, face-to-face and hybrid teaching contexts.

Keywords: Teaching; Education; Bioethics; Online Teaching; Pandemic.

${ }^{1}$ Universidade Federal de Alagoas, Maceió, Alagoas, Brasil.

${ }^{2}$ Universidade Federal Fluminense, Niterói, Rio de Janeiro, Brasil.

Editora-chefe: Daniela Chiesa.

Editora associada: Daniela Chiesa.

Recebido em 06/08/20; Aceito em 20/05/21.

Avaliado pelo processo de double blind review. 


\section{INTRODUÇÃO}

A Organização Mundial da Saúde (OMS) anunciou em março de 2020 a pandemia do novo coronavírus (coronavirus disease 2019 - Covid-19) e adotou inúmeras medidas na tentativa de diminuir a curva de contaminação da população mundial. Uma delas foi a recomendação do distanciamento social, seguida do isolamento social, que, entre outras repercussões, levou à necessidade de interrupção das atividades em diversos setores considerados não essenciais à sociedade. Consequentemente, todas as instituições educacionais tiveram suas aulas presenciais suspensas.

No Brasil, com a paralisação das atividades educacionais, - Ministério da Educação (MEC) lançou várias portarias, tendo a última, no 544, de 17 de junho de 2020, autorizado a substituição das disciplinas presenciais por aulas em ambientes digitais, com uso de tecnologias de informação e comunicação (TIC) ou outros meios convencionais'.

Indubitavelmente, apesar de o ensino remoto emergencial (ERE) e do ensino a distância (EaD) já serem uma realidade para algumas instituições de ensino superior (IES), isso trouxe, para inúmeras, vários desafios ${ }^{2}$. As universidades da área da saúde tiveram de se reinventar em relação ao processo educativo e, quase simultaneamente, têm discutido e executado as melhores opções para oferecer o acesso remoto e amenizar tanto a exclusão social quanto a digital, ambas agravadas com a pandemia.

A disciplina de bioética também teve de se reinventar, uma vez que, presencialmente, ela é caracterizada por discussões dialógicas e, frequentemente, abordada por meio de estratégias de metodologias ativas ou práticas morais. Como adequar o ensino virtual da bioética em tempos de pandemia sem agravar as inequidades? Essa pergunta direcionou nossa pesquisa, que resultou no seguinte objetivo: realizar uma reflexão propositiva para o ensino remoto em bioética em tempos de pandemia, pela ótica da educação em valores e da justiça social.

\section{INCLUSÃO SOCIODIGITAL E JUSTIÇA SOCIAL NA EDUCAÇÃO SUPERIOR EM TEMPOS DE PANDEMIA}

A discussão contemporânea sobre educação para a cidadania e em valores não pode ficar apartada do debate sobre a inclusão de uma diversidade cultural, racial, étnica, de gênero e social na universidade. Essa educação para a cidadania, baseada em pressupostos democráticos, igualitários e equitativos, vislumbra uma sociedade mais justa com escolhas pedagógicas e curriculares atreladas a valores que estão intrinsecamente relacionados à formação moral discente.

A interiorização das universidades públicas e a efetivação das políticas afirmativas do sistema de cotas trouxeram mudanças no perfil socioeconômico dos discentes de Medicina, que não podem ser ignoradas quando se planeja a educação on-line durante a pandemia. Um bom exemplo disso é a Universidade Estadual de Campinas (Unicamp) que desde 2005 implementou o Programa de Ação Afirmativa e Inclusão Social (Paais), com a finalidade de inclusão de estudantes do ensino médio das escolas públicas. Desde 2018, a instituição vem observando um maior percentual de ingresso de estudantes pardos e negros provenientes da rede pública ${ }^{3}$. Em 2019, ainda na mesma instituição, no curso de Medicina, verificou-se que esse percentual chegou a $85 \%$, alterando seu perfil socioeconômico, com predomínio nos estratos $\mathrm{C} 1$ e B273. Um estudo com estudantes de Medicina da Universidade Federal da Bahia (Ufba) apontou que $66,4 \%$ se autodeclaram negros e pardos, $44,4 \%$ estudaram a maior parte do ensino médio em escola pública e 17,6\% afirmaram ter renda familiar de até três salários mínimos ${ }^{4}$. Na Universidade Estadual de Londrina (UEL), uma análise do perfil socioeconômico de 2004 a 2012 concluiu que a renda familiar mais citada foi de sete a dez salários mínimos ${ }^{5}$. Porém, em 2013, registrou-se um maior número de ingressantes de Medicina com renda familiar de um a três salários mínimos ${ }^{5}$. Dados semelhantes foram encontrados na Universidade Federal do Pará (Ufpa), onde $30,9 \%$ dos estudantes de medicina declararam renda familiar de um a três salários mínimos, e 3,9\%, de até um salário mínimo ${ }^{6}$.

Ainda de acordo com a pesquisa da Unicamp, durante a pandemia, verificou-se que a maioria (80\%) dos estudantes acessava as aulas por meio de computador, notebook ou celular, somente $10 \%$ faziam isso exclusivamente por celular e $10 \%$ utilizavam tablet ${ }^{3}$. Os principais problemas encontrados de acessibilidade foram: internet instável e/ou acesso exclusivo por redes móveis, maior dificuldade para acompanhar atividades transmitidas por web conferências e meetings virtuais, e dificuldades para acessar as atividades em plataformas digitais e aplicativos de imagem ${ }^{3}$.

Tais dados, ainda que não generalizáveis, não excluem a relevância de observar esses estudantes que ingressaram em um curso elitizado e que não podem ser ignorados quando se planeja uma educação on-line, principalmente num período em que as desigualdades sociais tencionam uma exigência por escolhas mais equitativas, sobretudo nas instituições de educação que, em sua origem, não podem ser uma causa a mais de exclusão dos grupos já oprimidos socialmente. Todas elas, mas especialmente as de educação superior (objeto de nossa pesquisa), deverão estar comprometidas com o estabelecimento de uma cultura de inclusão e legitimação da diversidade, formando para a cidadania, na tentativa de minimizar desigualdades e aumentar a inclusão. 
A justiça não está exclusivamente relacionada à distribuição de bens entre os indivíduos (como distribuir aparelhos móveis a estudantes socialmente vulneráveis e concluir que essa ação isolada seria a solução para a questão do acesso remoto ao ensino), mas também às relações sociais e aos processos ${ }^{7}$. Os indivíduos são membros de grupos sociais diversos cujas experiências e oportunidades são influenciadas por suas filiações. E a interação dessas pessoas se baseia nas opressões que impactam os diferentes grupos sociais.

Nossa reflexão, na perspectiva de justiça na educação online, parte do pressuposto de que os indivíduos que fazem parte de grupos privilegiados ou oprimidos têm suas oportunidades de ensino e aprendizagem influenciadas por essa associação a um grupo social. Devem-se considerar suas especificidades e mecanismos de opressão que podem surgir nas escolhas pedagógicas e curriculares das instituições de ensino.

\section{A EDUCAÇÃO EM VALORES E O ENSINO DA BIOÉTICA}

Partimos do ponto de vista do ensino de uma bioética que se constrói na América Latina em contextos críticos de desigualdade e exclusão social, instigada a construir conhecimento e práticas que reúnem a filosofia, as ciências sociais, as ciências humanas e a saúde.

O campo da bioética ${ }^{8}$ abarca as dimensões sociais, econômicas, culturais e ambientais, em que se estabelecem discussões das mais diversas realidades, tais como preconceitos, exclusão, ecologia, entre outros, que são atravessados por conflitos morais. Um dos desafios atuais do ensino da bioética é contextualizar os conflitos morais já existentes e que foram potencializados devido à pandemia, de modo a contribuir para a discussão e a reflexão de temas fundamentais para a nossa realidade atual.
Para Puig, "a articulação de problemas morais significativos tratados com as ferramentas da inteligência moral e orientados pela cultura moral constitui uma excelente experiência de aprendizagem moral e de valores" ${ }^{\prime \prime}$. Dessa forma, podemos enfrentar de forma autônoma os conflitos de valor e as controvérsias de questões não resolvidas que perpassam a vida de indivíduos e dos grupos nas sociedades abertas, plurais e democráticas ${ }^{10}$.

A seguir descreveremos as capacidades que constroem o arcabouço da inteligência moral de acordo com Puig ${ }^{11,12}$.

Uma das reflexões propositivas deste artigo é que essas capacidades podem ser utilizadas como objetivos de aprendizagem nas disciplinas de bioética e planejadas e estimuladas nas estratégias pedagógicas ou nas práticas morais desenvolvidas em sala de aula, sejam estas presenciais ou virtuais.

As práticas morais para Puig ${ }^{10}$ podem ser consideradas como: "um curso de acontecimentos culturais estabelecido que permite enfrentar situações significativas, complexas ou conflitantes do ponto de vista moral". Ainda de acordo com o autor, uma prática moral é um meio de ensino e aprendizagem que problematiza situações habituais da vida; é uma situação pensada e disposta para aprender na prática social ${ }^{10}$. Mais adiante estabeleceremos sua correlação com o ERE e o EaD. Convém salientar que, na perspectiva de Puig ${ }^{12}$, não existe a possibilidade de construção moral sem a presença de problemas morais contextualizados.

\section{ESTRATÉGIAS DE ENSINO DA BIOÉTICA EM AMBIENTE VIRTUAL}

Com a pandemia, toda a discussão sobre o ERE e EaD, que levaria décadas, devido à sua premência, foi acelerada. Isso levou o MEC a autorizar a substituição e a adequação das

Quadro 1. Capacidades da inteligência moral de acordo com Puig.

\begin{tabular}{cl}
\hline Capacidades da inteligência moral & \multicolumn{1}{c}{ Características } \\
\hline Autoconhecimento & $\begin{array}{l}\text { Construir e valorar positivamente o próprio eu, conhecer a si mesmo, integrar a } \\
\text { experiência biográfica e projetá-la para o futuro. }\end{array}$ \\
Empatia & $\begin{array}{l}\text { Capacidade de adotar perspectivas sociais e de colocar-se no lugar do outro e do } \\
\text { reconhecimento dos sentimentos, das necessidades, das opiniões e dos argumentos dos } \\
\text { demais. }\end{array}$ \\
Julgamento moral & $\begin{array}{l}\text { Desenvolvimento da sensibilidade moral e da capacidade de pensar sobre os problemas } \\
\text { morais de maneira justa e solidária (cuidado com os demais). }\end{array}$ \\
Habilidades dialógicas & $\begin{array}{l}\text { Capacidade de mudar de opinião e de pensar nos pontos de vista dos outros } \\
\text { interlocutores com a intenção de entrar em entendimento. }\end{array}$ \\
Compreensão crítica & $\begin{array}{l}\text { Capacidade de adquirir informação e de comparar os diversos pontos de vista sobre a } \\
\text { realidade, com a finalidade de entendê-la melhor e comprometer-se a melhorá-la. }\end{array}$ \\
Autorregulação & $\begin{array}{l}\text { Buscar a coerência entre o julgamento e a ação moral, adquirir hábitos desejados e } \\
\text { construir voluntariamente o próprio caráter moral. }\end{array}$ \\
\hline
\end{tabular}

Fonte: Neves Júnior ${ }^{13}$ com base em Puig ${ }^{11,12}$. 
disciplinas presenciais por aulas que utilizem meios digitais, enquanto durar a situação de pandemia'.

Antes de dar continuidade à nossa reflexão propositiva sobre o ensino da bioética em ambiente virtual, precisamos diferenciar ERE de EaD. A modalidade EaD ocorre quando discentes e docentes não estão juntos no mesmo tempo ${ }^{14}$. Já o ERE refere-se à rápida mudança de panoramas, com necessidades e limitações diferentes, o que envolve uma alteração temporária decorrente de circunstâncias de crise ${ }^{14}$. Essa modalidade de ensino é considerada remota porque requer um distanciamento geográfico de alunos e professores de modo temporário e foi adotada em diferentes níveis de ensino por instituições educacionais do mundo todo, para que as atividades escolares não fossem interrompidas em meio à pandemia ${ }^{15}$.

Como parte da proposição reflexiva, podemos utilizar, nas disciplinas de bioética, práticas morais que se relacionam com as seguintes capacidades: o autoconhecimento, a empatia, o julgamento moral, as habilidades dialógicas, a compreensão crítica e a autorregulação.

No ERE, as aulas virtuais podem ocorrer no que se chama de tempo síncrono (podendo seguir os princípios do ensino presencial), utilizando para isso videoaulas, aulas expositivas por sistema de webconferência (por exemplo, Zoom, Google Meet, RPN etc.), chats e bate-papos ${ }^{15,16,17}$. Já as atividades do EaD podem ocorrer também de forma assíncrona durante a semana no ambiente virtual de aprendizagem (AVA), como Moodle, Teleduc, Blackboard ${ }^{15,17}$. É de suma importância destacar que todas as atividades, sejam do ERE ou do EaD, devem ser elaboradas e planejadas vinculando a prática moral, os conteúdos didáticos e os objetivos de aprendizagem desejados a fim de facilitar a formação moral.

Segundo Maluf e Garrafa"18, "uma das dificuldades pedagógicas para o desenvolvimento da bioética como disciplina é a construção de seus conteúdos, como ela deve ser estruturada, como deve ser definida em termos de domínio das concepções teórico-práticas e de seus objetivos".

Sendo assim, uma outra reflexão propositiva é tentar contextualizar os conteúdos didáticos. Para isso, mesclamos algumas sugestões de temas propostos pelo Programa de Base de Estudos sobre Bioética (Core Curriculum) desenvolvido pela Organização das Nações Unidas para a Educação, a Ciência e a Cultura (Unesco) ${ }^{19}$, pelo Grupo de Ensino e Pesquisa em Bioética (GEPBio) ${ }^{20}$, bem como outros que avaliamos como pertinentes para discussão nas disciplinas de bioética. Como resultado, além das questões que já eram discutidas no currículo, listamos: dignidade humana; direitos humanos; respeito à vulnerabilidade humana e à integridade pessoal; igualdade, equidade e justiça social e suas relações diretas com os seguintes conteúdos: raça, gênero, população de rua, população indígenas e quilombolas, favelas, comunidade LGBT, em especial as pessoas transgêneros; pesquisa envolvendo seres humanos; alocação de recursos em saúde; telemedicina; judicialização em saúde; proteção do meio ambiente; a biosfera e a biodiversidade; saneamento básico ${ }^{19,20}$.

Algumas disciplinas já realizavam essas discussões em seus conteúdos programáticos, precisa-se agora é problematizar nos cursos de Medicina como avançar a discussão para a inserção dos diversos espectros da diversidade humana biológica, subjetiva, étnico-racial, de gênero, orientação sexual, socioeconômica, política, ambiental, cultural, ética - previstos nas Diretrizes Curriculares Nacionais (DCN) ${ }^{21}$. Faz-se necessário aprimorar esse debate sobre a responsabilidade social das escolas médicas, não somente neste período de pandemia, mas também no sentido da promoção da justiça social para redução de iniquidades ${ }^{21}$. Devem-se contextualizar todos esses temas no momento atual, bem como adequar as discussões de conteúdos já existentes antes da pandemia nos currículos, como: o início e o fim da vida humana, princípios da bioética, sigilo e confidencialidade, e relação profissional com o paciente.

Além dessas tentativas de readequações de conteúdo e objetivos de ensino-aprendizagem, faz-se necessário discutir estratégias de inclusão sociodigital com o corpo docente e com as IES. Cabe ressaltar que essas medidas não podem ser pontuais e entendidas como uma solução para a questão do acesso remoto ao ensino; é fundamental um acompanhamento próximo e contínuo dos estudantes identificados como em situação de vulnerabilidade social, para que se possa oferecer a eles um suporte pedagógico, emocional, socioeconômico e digital.

\section{CONSIDERAÇÕES FINAIS}

A pandemia da Covid-19, devido ao seu caráter de urgência, acelerou a inserção do ensino remoto na educação, e este, ao mesmo tempo que proporcionou avanços aos currículos da área da saúde com a inclusão de TIC, descortinou muitas incertezas. Para a disciplina de bioética, não foi diferente, não somente nos questionamentos na educação sobre as desigualdades sociodigitais, econômicas e de saúde, como também na aprendizagem, devido à perda do contato e das discussões presenciais, essenciais para a construção da personalidade moral. O desafio lançado é incorporar os objetivos de aprendizagem consonantes às práticas morais e conteúdos nas disciplinas de bioética e manter as habilidades dialógicas e participativas no ambiente virtual.

Esperamos que nossas reflexões propositivas possam contribuir para novas reflexões sobre o ensino da bioética, a fim de que possamos enxergar os problemas e dilemas bioéticos 
exponencialmente aumentados na sociedade neste momento de pandemia, sob as lentes da justiça social, e, com isso, auxiliar no desenvolvimento moral de nossos estudantes.

\section{CONTRIBUIÇÃO DOS AUTORES}

Waldemar Antônio das Neves Júnior foi responsável pela conceituação do trabalho, pela curadoria de dados, pela análise formal, pela investigação, pela metodologia e pela redação do manuscrito. Lumaira Maria Nascimento Silva da Rocha Marques e Michelle Cecille Bandeira Teixeira participaram da investigação, da metodologia, redação do artigo e da revisão de conteúdo.

\section{CONFLITO DE INTERESSES}

Declaramos não haver conflito de interesses.

\section{FINANCIAMENTO}

Declaramos não haver financiamento.

\section{REFERÊNCIAS}

1. Brasil. Portaria $n^{\circ} 544$, de 16 junho de 2020. Dispõe sobre a substituição das aulas presenciais por aulas em meios digitais, enquanto durar a situação de pandemia do novo coronavírus - Covid-19 [access in 17 jun 2020]. Available from: http://www.in.gov.br/en/web/dou/-/portaria-n544-de-16-de-junho-de-2020-26192487.

2. Martelli Júnior H, Machado RA, Swerts MSO, Martelli DRB, Caldeira AP. The works of Hercules and Covid-19 lessons for medical education. Rev Bras Educ Med. 2020;44(3):e084 [access in 28 jul 2020]. Available from: https://www.scielo.br/scielo.php?script=sci_arttext\&pid=S0100$55022020000300603 \& \operatorname{lng}=$ pt\&nrm=iso\&tlng=en.

3. Appenzeller S, Menezes FH, Santos GG, Padilha RF, Graça HS, Bragança JF. Novos tempos, novos desafios: estratégias para equidade de acesso ao ensino remoto emergencial. Rev Bras Educ Med. 2020;44(sup.1):e0155 [access in $20 \mathrm{dec}$ 2020]. Available from: https://www.scielo.br/pdf/rbem/ v44s1/1981-5271-rbem-44-s1-e155.pdf.

4. Veras RM, Fernandez CC, Feitosa CCM, Fernandes S. Perfil socioeconômico e expectativa de carreira dos estudantes de Medicina da Universidade Federal da Bahia. Rev Bras Educ Med. 2020;44(2):e056 [access in 3 oct 2020]. Available from: https://www.scielo.br/pdf/rbem/v44n2/1981-5271rbem-44-02-e056.pdf.

5. Guariente SMM, Guariente MHDM, Moraes A. Perfil sociodemográfico do estudante ingressante no curso de graduação em medicina de 2004 a 2013: análise documental. Rev Méd Minas Gerais. 2020;30:e-30102 [access in 3 oct 2020]. Available from: http://rmmg.org/artigo/detalhes/2664.

6. Rego RM, Marques NA, Monteiro PC, Oliveira CLB, Lins NAA, Caldas CAM. O perfil atual do estudante de medicina e sua repercussão na vivência do curso. Para Res Med J. 2018;2(1-4):e05 [access in 3 oct 2020]. Available from: https://prmjournal.org/article/10.4322/prmj.2018.005/pdf/prmjournal-2-14-e05.pdf.
7. Young IM. Justice and the politics of difference. Princeton: Princeton University Press; 1990.

8. Vicente AL. Sobre la bioética iberoamericana: una paradoja. Humanidad Med. 2014;14(3):570-88.

9. Arantes V, Araújo UF, Silva MAM. Josep Maria Puig: uma vida dedicada à educação em valores. Educ Pesqui. 2019;45:e201945002001 [access in 20 dec 2020]. Available from: https://www.revistas.usp.br/ep/article/ view/162695/156537.

10. Puig JM. Práticas morais: uma abordagem sociocultural da educação moral. São Paulo: Moderna; 2004. 200 pp. p. 62.

11. Puig JM, Araújo UF, Arantes VA. Educação em valores: pontos e contrapontos. São Paulo: Summus; 2007.

12. Puig JM. Construcción de la personalidad moral. Barcelona: Paidós; 1996.

13. Neves Júnior WA. A educação em valores e as práticas morais nas disciplinas de bioética de faculdades de medicina no Estado de São Paulo [tese]. Rio de Janeiro: Fundação Oswaldo Cruz; 2016. 143pp. p.43.

14. Hodges C, Moore S, Lockee B, Trust T, Bond A. The Difference between emergency remote teaching and online learning. Educause review. 2020 [access in 11 jul 2020]. Available from: https://er.educause.edu/ articles/2020/3/the-difference-between-emergency-remote-teachingand-online-learning.

15. Cani JB, Sandrini EGC, Soares GM, Scalzer K. Educação e Covid-19: a arte de reinventar a escola mediando a aprendizagem "prioritariamente" pelas TDIC. Revista IFES Ciência. 2020;6(1):23-39 [access in 28 jul 2020]. Available from: https://www.researchgate.net/publication/342223833 EDUCACAO_E_COVID-19_A_ARTE_DE_REINVENTAR_A_ESCOLA MEDIANDO_A_APRENDIZAGEM_prioritariamente_PELAS_TDIC.

16. Behar PA, Schneider D. Modelos pedagógicos e Competências em Educação a Distância: a construção do MP - CompEAD. Revista de Educação Pública. 2016;25(59/2):504-24 [access in 11 jul 2020]. Available from: https://periodicoscientificos.ufmt.br/ojs/index.php/educacaopublica/ article/view/3832.

17. Torres RM, Liu PMF. Guia prático para o uso de plataformas virtuais no ensino remoto. Belo Horizonte: UFMG; 2020 [access in 28 jul 2020]. Available from: https://www.medicina.ufmg.br/wp-content/uploads/ sites/7/2020/07/E-book-Guia-pr\%C3\%A1tico-plataformas-virtuais-3.pdf.

18. Maluf F, Garrafa V. O Core Curriculum da Unesco como base para formação em bioética. Rev Bras Educ Med. 2015;39(3):456-62. p.460.

19. Organização das Nações Unidas para a Educação, a Ciência e a Cultura. Programa de Base de Estudos sobre Bioética da Unesco. Unesco; 2015 [access in 11 jul 2020]. Available from: http://www.unesco.org/new/ fileadmin/MULTIMEDIA/FIELD/Montevideo/pdf/Bioet-CoreCurriculumPT-Parte1.pdf.

20. Grupo de Ensino e Pesquisa em Bioética. Temas bioéticos em tempos de pandemia [livro online]. Belo Horizonte: Newton; 2020 [access in 11 jul 2020]. Available from: http://www.sbbioetica.org.br/Noticia/717/ GEPBio-lanca-e-disponibiliza-a-Cartilha-Temas-Bioeticos-em-tempos-depandemia-Faca-o-download-e-consulte-o-texto-na-integra.

21. Raimondi GA, Tourinho FSV, Souza FGR, Pereira DVR, Oliveira DOPS, Rosa LM. Análise crítica das DCN à luz das diversidades: educação médica e pandemia da Covid-19. Rev Bras Educ Med. 2020;44(sup 1):e0135 [access in $20 \mathrm{dec}$ 2020]. Available from: https://www.scielo.br/scielo. php?script=sci_arttext\&pid=S0100-55022020000500801\&lng=pt\&nrm= so\&tlng=pt. 